\title{
Anti-tumor effects of telomelysin for head and neck squamous cell carcinoma
}

\author{
KYOKO FUJITA, MACHIKO KIMURA, NORIO KONDO, ATSUKO SAKAKIBARA, \\ DAISUKE SANO, YUKARI ISHIGURO and MAMORU TSUKUDA

\begin{abstract}
Department of Biology and Function in Head and Neck, Yokohama City University
\end{abstract} \\ Graduate School of Medicine, 3-9 Fukuura, Kanazawa-ku, Yokohama 236-0004, Japan
}

Received May 27, 2008; Accepted September 3, 2008

DOI: $10.3892 /$ or_00000153

\begin{abstract}
Telomelysin is a telomerase-specific replicationcompetent adenovirus with telomerase reverse transcriptase (hTERT) promoter, which has shown strong anti-tumor effects on a variety of human cancer cells. Human head and neck squamous cell carcinoma (HNSCC) cell lines and a murine HNSCC (NR-S1) model were used to investigate whether telomelysin (OBP-301) had a therapeutic efficacy for HNSCC. We examined the cell killing effects of telomelysin and the induction of tumor cell apoptosis by telomelysin in vitro. Based on these data, we examined whether telomelysin therapy produced therapeutic benefits in vivo. The results demonstrated that the treatment of telomelysin led to significant tumor regression on the side with subcutaneous NR-S1 tumor. We first confirmed the direct anti-tumor effect of intratumoral telomelysin injections in a murine HNSCC model. Further analyses of the augmented anti-tumor effects revealed that telomelysin increased the source of tumor antigens for immune cells, resulting in the induction of $\mathrm{CD}^{+}$ and $\mathrm{CD}^{+} \mathrm{T}$ cells responsible for the in vivo tumor regression of treated and untreated tumors. Subsequently, an elevated IFN- $\gamma$ production of spleen cells was observed in mice treated with telomelysin. These results raise the possibility that telomelysin enhances the immune response in addition to its direct tumor cell killing activity. These findings suggest that telomelysin is a potent agent for the treatment of HNSCC patients with multiple metastases.
\end{abstract}

\section{Introduction}

The incidence of head and neck squamous cell carcinoma (HNSCC) is $\sim 500,000$ cases annually worldwide (1).

Correspondence to: Dr Kyoko Fujita, Department of Biology and Function in Head and Neck, Yokohama City University Graduate School of Medicine, 3-9 Fukuura, Kanazawa-ku, Yokohama 236-0004, Japan

E-mail: t056043b@yokohama-cu.ac.jp

Key words: telomelysin (OBP-301), adenovirus, apoptosis, immunotherapy, head and neck squamous cell carcinoma
Combined treatments including surgery, chemotherapy and radiotherapy prolong the survival of advanced cases with HNSCC, but the treatment benefit has been typically temporary in advanced disease (2). Therefore, new approaches are necessary to provide more effective treatment modalities, e.g. molecular-targeted therapy, gene therapy and immunotherapy, against advanced disease.

Telomerase is a rib nucleoprotein complex responsible for the complete replication of chromosomal ends, whose activation is considered to be a crucial step not only in cell proliferation but also in carcinogenesis (3-6). Previous studies have demonstrated a high telomerase activity in $>85 \%$ of human cancers (4), but in only a few normal somatic cells (5). Human telomerase reverse transcriptase (hTERT) positively regulates telomerase at the transcriptional level and shows a silectively high activity in growing neoplastic tissues and cells. Therefore, it seems reasonable to use hTERT promoter as a cancer-specific promoter. Telomelysin (OBP-301) is a telomerase-specific replication-competent adenovirus that induces selective E1 expression and exclusively kills human cancer cells (7-10).

Deng et al reported that the E1A gene showed induction apoptosis (11). It has also been reported that the uptake of antigens from apoptotic cells induces cytotoxic T-lymphocytes (CTLs) which are recognized as critical mediators of anti-tumor immunity (12). Endo et al reported that telomelysinmediated oncolysis stimulated CTL activity (13). These findings led us to examine whether tumor cells killed by telomelysin infection were able to stimulate an immune response in vivo by antigens of the apoptotic cells induced by telomelysin. Therefore, we analyzed the anti-tumor activity of telomelysin on human HNSCC cell lines and anti-tumor mechanisms using a murine HNSCC cell line in vitro and in vivo. The anti-tumor effects of telomelysin on HNSCC have yet to be reported.

\section{Materials and methods}

Human cell lines and culture conditions. Human HNSCC cell lines were examined in this study. The primary sites of these cell lines were the oral floor (YCU-OR891), hypopharynx (YCU-H891), mesopharynx (YCU-M862, YCU-M911 and KCC-M871), larynx (KCC-L871 and YCU-L891), tongue (YCU-T891, YCU-T892, KCC-T871, KCC-T873 and HSC-3), 
Table I. Antiproliferative activity of telomelysin. ${ }^{\mathrm{a}}$

\begin{tabular}{|c|c|c|c|c|}
\hline \multirow[b]{2}{*}{ HNSCC cell line } & \multirow[b]{2}{*}{ Primary sites } & \multicolumn{3}{|c|}{ Telomelysin $\mathrm{ID}_{50}(\mathrm{MOI})$} \\
\hline & & Day 3 & Day 5 & Day 7 \\
\hline YCU-OR891 & Oral floor & 13875 & 3549 & 3077 \\
\hline YCU-H891 & Hypopharynx & 2886 & 2931 & 279 \\
\hline YCU-M862 & Mesopharynx & 4120 & 3510 & 3186 \\
\hline YCU-M911 & Mesopharynx & 3189 & 2179 & 2819 \\
\hline KCC-M871 & Mesopharynx & 31041 & 4661 & 4585 \\
\hline KCC-L871 & Larynx & 12389 & 2962 & 2894 \\
\hline KCC-L891 & Larynx & 4099 & 3223 & 3015 \\
\hline YCU-T891 & Tongue & 3570 & 2736 & 2918 \\
\hline YCU-T892 & Tongue & 4776 & 2865 & 2788 \\
\hline KCC-T871 & Tongue & 13787 & 3744 & 3075 \\
\hline KCC-T873 & Tongue & 4523 & 3455 & 3050 \\
\hline HSC-3 & Tongue & 16814 & 3811 & 3065 \\
\hline КСС-ТСМ901 & Lung metastasis (tongue) & 3365 & 3115 & 314 \\
\hline КСС-ТСМ902 & Lung metastasis (tongue) & 3322 & 2161 & 282 \\
\hline КСС-ТСМ903 & Lung metastasis (tongue) & 4684 & 3560 & 3055 \\
\hline KCC-MS871 & Maxillary sinus & 44057 & 3909 & 3243 \\
\hline KCC-MS861 & Maxillary sinus & 3311 & 2791 & 2208 \\
\hline
\end{tabular}

${ }^{a}$ In the proliferation assay described in Materials and methods, tumor cells were exposed to telomelysin for $24 \mathrm{~h}$. Data are expressed as $\mathrm{ID}_{50}$, the telomelysin quantity (MOI) that causes $50 \%$ inhibition of cell proliferation.

lung metastasis of tongue cancer (KCC-TCM901, KCCTCM902 and KCC-TCM902) and maxillary sinus (KCCMS871 and YCU-MS861). These cell lines were maintained in RPMI-1640 medium (Life Technologies Inc., Tokyo, Japan) supplemented with $10 \%$ fetal bovine serum, 2 mmol/l L-glutamine, $100 \mathrm{U} / \mathrm{ml}$ penicillin and $100 \mu \mathrm{g} / \mathrm{ml}$ streptomycin. The cells were incubated at $37^{\circ} \mathrm{C}$ in an atmosphere containing $5 \% \mathrm{CO}_{2}$.

Mice and cell line. Four-week-old female $\mathrm{C} 3 \mathrm{H}\left(\mathrm{H}-2 \mathrm{k}^{\mathrm{k}}\right)$ mice were purchased from Oriental Yeast, Tokyo, Japan. They were maintained in the specified pathogen-free conditions and used after approval by the Animal Care and Use Committee of Yokohama City University School of Medicine. A murine squamous cell carcinoma cell line (NR-S1) originating from the bucal mucosa was cultured in DMEM supplemented with $10 \%$ fetal bovine serum, $2 \mathrm{mmol} / \mathrm{l} \mathrm{L-glutamine,} 100 \mathrm{U} / \mathrm{ml}$ penicillin and $100 \mu \mathrm{g} / \mathrm{ml}$ streptomycin.

Adenovirus. We used the recombinant replication-selective, tumor-specific adenovirus vector telomelysin (OBP-301), in which the hTERT promoter element drives the expression of E1A and E1B genes linked with an internal ribosome entry site $(7,8)$.

In vitro proliferation assays. MTT assay was performed to measure cell viability using human HNSCC cell lines and a mouse HNSCC cell line. These cells were plated in 96-well U-bottomed plates (Falcon, Becton-Dickinson Labware, Lincoln Park, NJ, USA) at a concentration of $1 \times 10^{3}$ cells/ well. Plates were allowed to incubate for $24 \mathrm{~h}$ prior to drug treatment. After a 3-, 5- and 7-day exposure to drugs, the MTT assay was carried out with Tetra Color One (Seigaku Co., Ltd., Tokyo, Japan). Relative growth inhibition was calculated compared to vehicle-treated control cells, and $\mathrm{ID}_{50}$ values acted as the drug concentration showing $50 \%$ survival.

Annexin $V$ binding assay. To evaluate apoptosis in NR-S1 cells, an Annexin V binding assay was performed. Tumor cells were double stained with FITC-conjugated Annexin V and propidium iodide (Roche). Cells undergoing early apoptosis were determined as the percentage of Annexin V-positive propidium iodide-negative cells by FACScan with a Cell Quest 1.0 software package (Becton-Dickinson).

In vivo anti-tumor activity studies. Female $\mathrm{C} 3 \mathrm{H} / \mathrm{HeJ}$ mice, 4 weeks old, were obtained from Oriental Yeast. The mice were maintained in a laminar flow room with constant temperature and humidity. Suspensions of $100 \mu 1$ of NR-S1 cells (final concentration: $5 \times 10^{6}$ cells $/ \mathrm{ml}$ ) were injected subcutaneously into the two sides of the mice. Tumor-bearing mice were randomized $(n=7)$ when the mean tumor volume was $\sim 100$ $\mathrm{mm}^{3}$. Each group was closely matched before treatment, which began 10 days after cell transplantation. The mice were randomly assigned to the treatment groups with an intratumoral injection of PBS (control) or telomelysin in $100 \mu 1$ buffer. As for the telomelysin administration, the leftside tumors were intratumorally injected with either doses of a single administration or three separate administrations of $10^{8}$ plaque-forming units (PFU) per tumor. Tumor diameters 


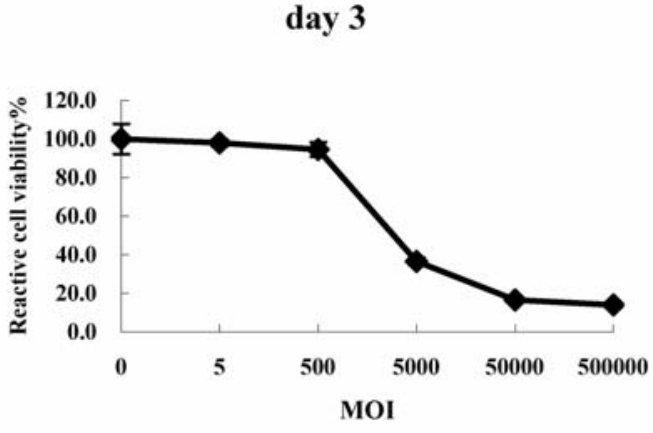

day 5

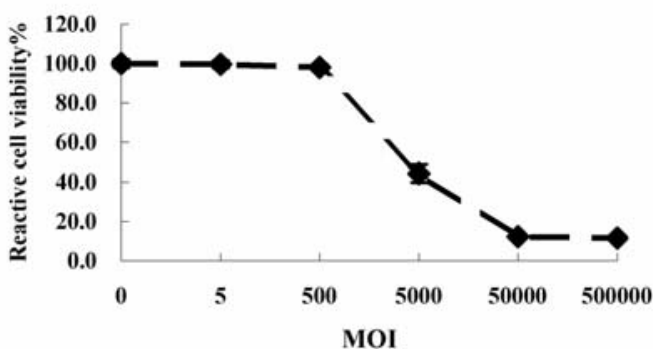

day 7

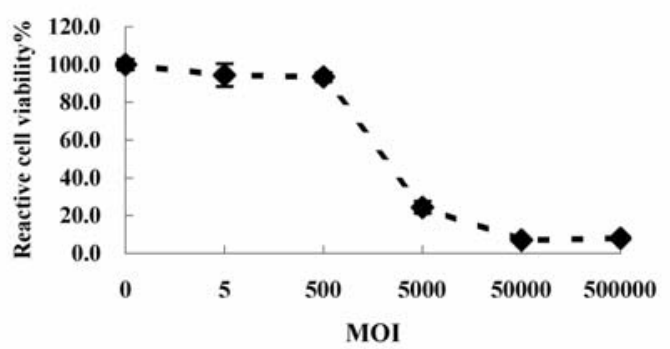

Figure 1. In vitro cell killing effect of telomelysin on NR-S1 murine SCC cells. NR-S1 cells were infected with telomelysin at the indicated multiplicity of infection (MOI) values, and surviving cells were quantitated over 3, 5 and 7 days after infection by MTT assay. Error bars indicate SE.

in the control and treated groups were measured weekly with a Vernier caliper. Tumor volume (V) was determined by the equation: $\mathrm{V}=\mathrm{ab}^{2} / 2$ ( $\mathrm{a}$, length and $\mathrm{b}$, width).

$I F N-\gamma$ assay. Spleen cells $\left(1 \times 10^{6} / \mathrm{ml}\right)$ isolated from telomelysin-treated (triple administration) and control mice were added to 6-well plates and stimulated with mitomycintreated NR-S1 HNSCC cells $\left(1 \times 10^{5} / \mathrm{ml}\right)$. Supernatants were collected 5 days later, centrifuged ( $1500 \mathrm{x}$ g for $15 \mathrm{~min}$ ), and stored at $-80^{\circ} \mathrm{C}$. Levels of IFN- $\gamma$ were assessed by ELISA (Bioscience).

Immunohistochemical assay. The mice were sacrificed on day 7 after being treated three times with PBS or telomelysin. Tumors were resected and stored in Tissue-Tek OCT compound (Sakura Finetechnical Co. Ltd., Tokyo, Japan) at $-80^{\circ} \mathrm{C}$ until immunohistochemical analysis. We immunohistochemically evaluated the explants of tumor-bearing mice for the presence of CD4 (Santa Cruz Biotechnology, Inc., CA, USA) or CD8 (Chemicon International, Inc., CA, USA) T cells

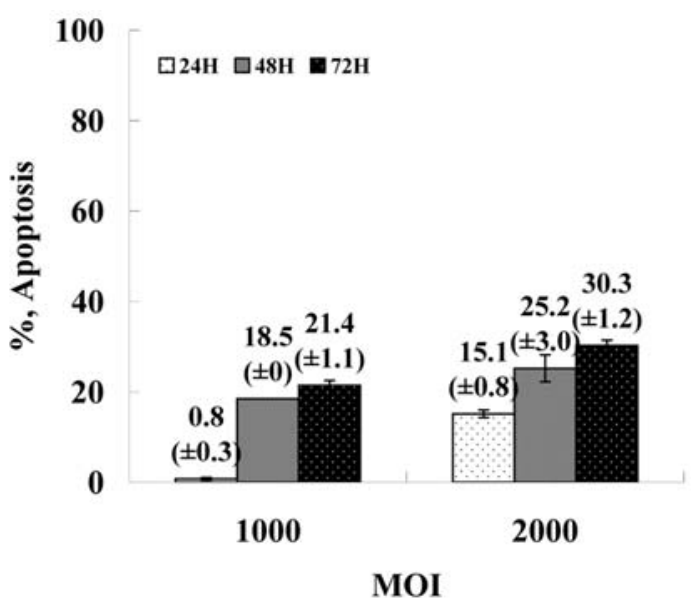

Figure 2. Telomelysin-induced apoptosis of NR-S1 cells. NR-S1 cells were treated with various concentrations of telomelysin in vitro. Tumor cell apoptosis harvested after a 24 and 48 h coincubation with telomelysin was assessed by Annexin V/propidium iodide staining and FACScan analysis. Error bars indicate SE.

and CD56 (Santa Cruz Biotechnology, Inc.) after streptavidin peroxidose immunization.

Statistical analysis. For statistical analyses of in vivo antitumor activity, IFN- $\gamma$ assay and immunohistological analysis, we used Student's paired t-test. $\mathrm{P}<0.01$ was considered significant.

\section{Results}

Antiproliferative effects of telomelysin on human HNSCC lines. We initially determined the antiproliferative effects of telomelysin on HNSCC lines. Cells were cultured with media alone (control) or media containing telomelysin (0-5000 multiplicity of infection, MOI). As shown in Table I, HNSCC lines were sensitive to telomelysin. The $\mathrm{ID}_{50}$ of telomelysin on day 7 ranged between 279 and 4585 MOI.

Antiproliferative effects of telomelysin on NR-S1 cells. The cell killing effect of different concentrations of telomelysin on the NR-S1 cell line was determined. As illustrated in Fig. 1, significant growth inhibitory effects were observed on days 3,5 and 7 following telomelysin treatment at $>500$ MOI.

Analysis of apoptosis in NR-S1 cells induced by telomelysin in vitro. To determine the apoptotic rates in NR-S1 cells treated with telomelysin, we subtracted the percentage of spontaneous apoptosis in untreated tumor cell cultures from telomelysin-induced apoptosis values. In the Annexin V binding assay the apoptotic rate determined as the percentage of Annexin V-positive propidium iodide-negative cells treated with telomelysin for $72 \mathrm{~h}$ was $21.4 \pm 1.1$ and $30.3 \pm 1.2 \%$ at the concentrations of 1000 and 2000 MOI, respectively (Fig. 2).

In vivo study of the anti-tumor effects of telomelysin on NR-S1 xenografts. We investigated the in vivo anti-tumor activity of telomelysin in the NR-S1 xenografts. Tumor growth was significantly inhibited by treatment with telomelysin on the 
A

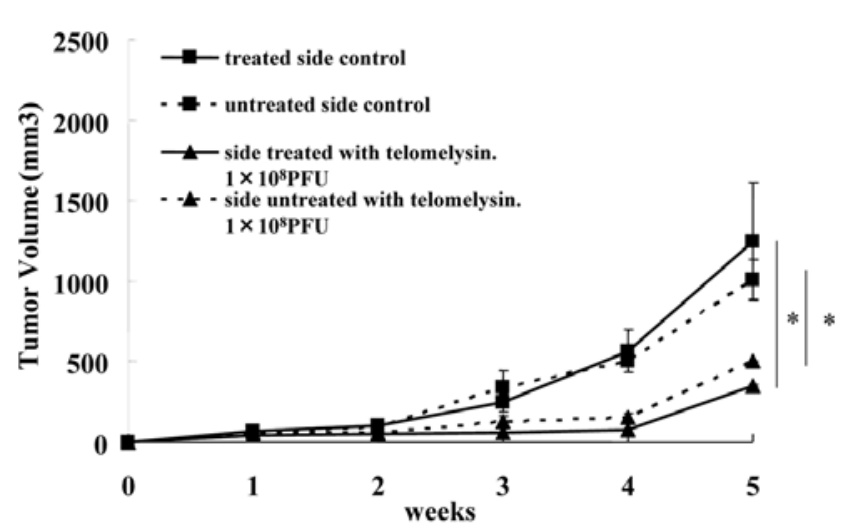

B

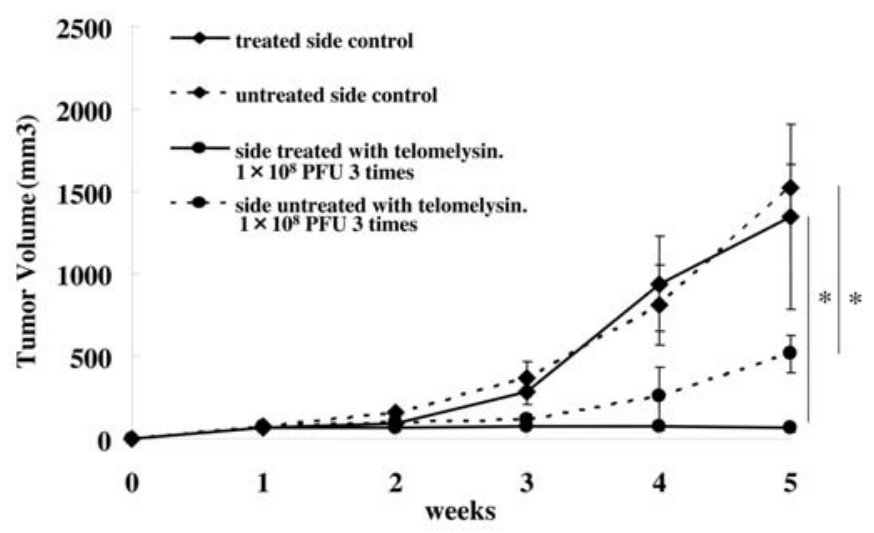

Figure 3. (A and B) Effects of telomelysin on tumor growth on the two sides of the HNSCC xenograft model. (A) One- and (B) three-time telomelysin administration of $1 \times 10^{8} \mathrm{PFU}$. Antitumor potential of telomelysin was significantly stronger than the control in the murine SCC model. Error bar, SE ( $=7$ mice per group) and ${ }^{*} \mathrm{P}<0.01$ versus control.

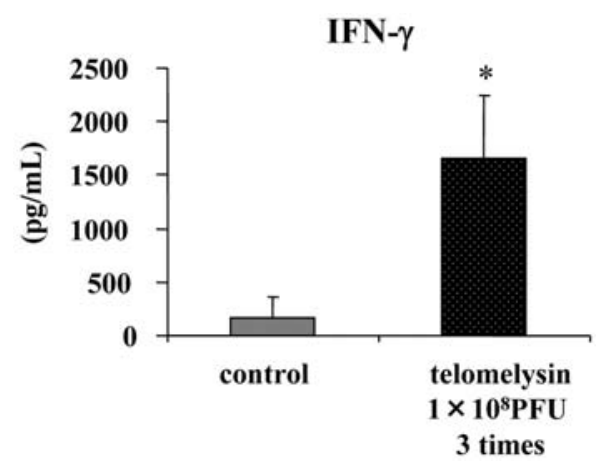

Figure 4. Effects of telomelysin on IFN- $\gamma$ secretion by spleen cells. The cells were stimulated with mitomycin-treated NR-S1 cells and incubated for 5 days. The supernatants were collected and evaluated by ELISA for the production of IFN- $\gamma$. Columns, mean of a triplicate sample; error bars indicate SE and ${ }^{*} \mathrm{P}<0.01$ versus control.

treated side, and tumor reduction was observed in the untreated side of the group receiving telomelysin injection compared to the control group $(\mathrm{P}<0.01$; Fig. 3A and $\mathrm{B})$. The telomelysin treatment courses of $10^{8} \mathrm{PFU} /$ day, i.e., once or three times, revealed a dose-dependent inhibition of the tumor growth on the treated side of mice with tumor xenograft.

IFN- $\gamma$ production in spleen cells of mice treated with telomelysin. To evaluate the immunological response to the telomelysin treatment, IFN- $\gamma$ production was assessed in cultures of spleen cells isolated from the control group, and in telomelysin-treated cultures. IFN- $\gamma$ was measured in the supernatants of spleen cells which were stimulated with mitomycin-treated NR-S1 (Fig. 4). Spleen cells isolated from mice treated with telomelysin released a significantly higher level of IFN- $\gamma$ compared to that of spleen cells from the control group.

Immunohistochemical analysis. The infiltration of $\mathrm{CD}^{+}$or $\mathrm{CD}^{+} \mathrm{T}$-lymphocytes in tumors receiving telomelysin injections on the treated and untreated sides was significantly enhanced compared with that observed in the control group. There was almost no infiltration of $\mathrm{CD}^{2} 6^{+}$cells in either the treated or control groups (Fig. 5A and B).

\section{Discussion}

The successful multidisciplinary treatment of advanced head and neck cancer is a significant issue. Neither cytotoxic therapy nor immunotherapy alone or in combination with definitive treatments, i.e., surgery and/or radiotherapy, can successfully resolve this issue. Different treatment modalities may provide an improvement in the outcome of advanced cases. In the present study, we evaluated the potential utility of the intratumoral administration of telomelysin, an anticancer virus.

Telomelysin has been shown to be effective against human cancers $(7,8,10)$. This virus was genetically designed to replicate under the control of the hTERT promoter specifically in tumor cells causing specific 'oncolysis'. Therefore, telomelysin does not cause significant toxicity in normal human cells and tissues $(7,8,10)$.

This is the first study on the anti-tumor effects of telomelysin on HNSCC. The present results demonstrated that telomelysin has a strong anti-tumor effect against human HNSCC cells in vitro and against a murine HNSCC cell line in vitro and in vivo. Kishimoto et al reported that the reactive hTERT mRNA expression in HNSCC cell lines was detected by real-time RT-PCR analysis (14). Therefore, telomelysin showed killing activity against HNSCC in in vitro and in vivo studies. The cell growth inhibition in vitro and the mouse model with tumor xenografts treated with telomelysin $\left(10^{8}\right.$ PFU/day, once or three times) showed a dose-dependent inhibition of tumor growth on the treated side. The intratumoral administration of telomelysin is a rational therapy for HNSCC patients because most patients have visible tumors in the head and neck regions.

Endo et al (13) reported that telomelysin replication produced the endogenous danger-signaling molecule, uric 
A

treated side CD8

untreated side CD8

treated side CD4

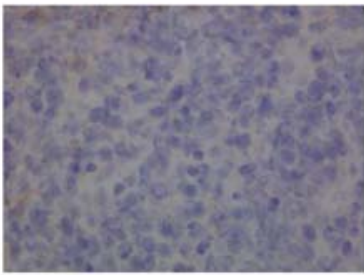

untreated side CD4
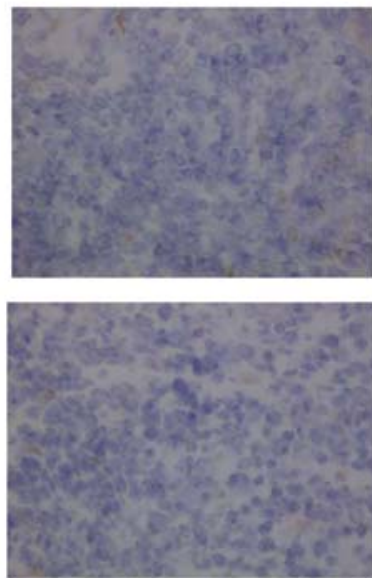

telomelysin

$10^{8}$ PFU 3 times
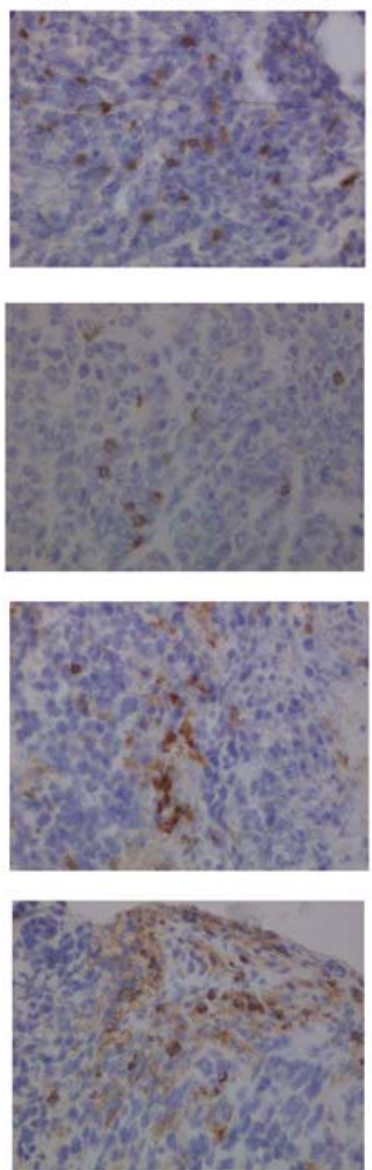

$\mathbf{B}$

CD4
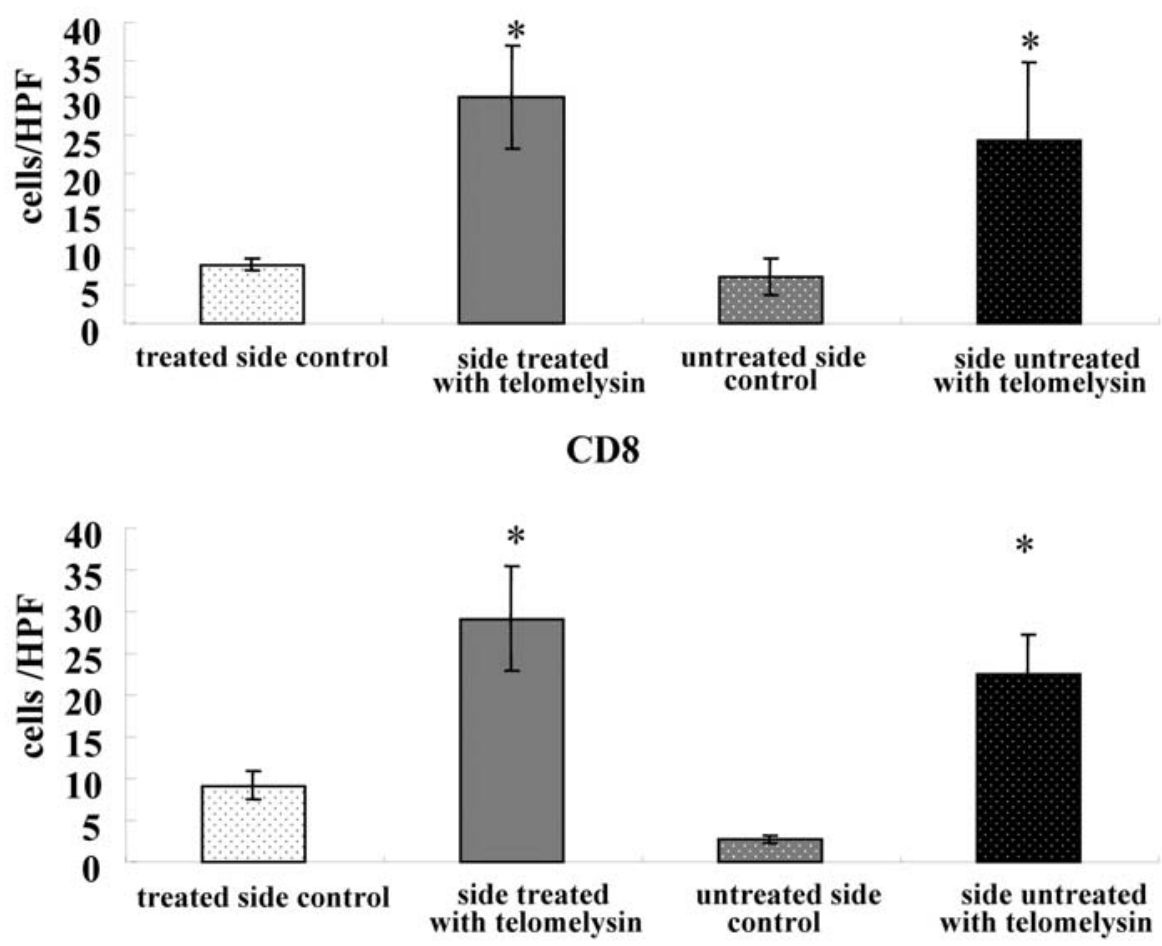

Figure 5. (A and B) Seven days after telomelysin treatment, control and treated mice were sacrificed. Tumors from the treated and untreated sides were sectioned and stained for CD4 and CD8 T-cell infiltration. Magnification, x400. Five fields per histological section were included in the analysis. Error bars indicate $\mathrm{SE}$ and ${ }^{*} \mathrm{P}<0.01$ versus control. 
acid, in infected human tumor cells, which in turn stimulated dendritic cells (DCs) to produce IFN- $\gamma$ and IL-12 in vitro. We demonstrated that telomelysin induced the apoptotic cell death of NR-S1 cells and that treatment with telomelysin led to tumor regression in treated and untreated tumors in an immunocompetent murine SCC model. In tumors on the untreated side, we found that telomelysin increased apoptotic tumor cells and induced $\mathrm{CD}^{+}$and $\mathrm{CD}^{+} \mathrm{T}$ cells responsible for the in vivo tumor regression of treated and untreated tumors. Subsequently, elevated IFN- $\gamma$ production of spleen cells in mice treated with telomelysin was observed.

Recent studies have shown that telomelysin is effective not only as a direct cytotoxic agent but also as an immunostimulatory agent which can induce specific CTL for the remaining antigen-bearing tumor cells (13). Nicolas et al have debated on whether necrotic or apoptotic cells can stimulate DCs to cross-present cell-derived peptides (15). Loading of DCs with tumor antigens can be facilitated by the selective use of a stress regimen that promotes apoptosis, which raises the possibility of a receptor-mediated uptake of apoptotic materials $(12,15,16)$. Therefore, telomelysin may increase the source of tumor antigens for immune cells, and accelerate or sustain the anti-tumor immunity, resulting in therapeutic effects. Our data support this hypothesis.

We investigated the anti-tumor effect of telomelysin combined with DCs (data not shown). The results demonstrated that treatment with telomelysin combined with the injection of DCs led to complete tumor regression, in contrast to only partial eradication of the tumors with DCs alone. This approach is based on previous findings that DCs are able to take up apoptosis of tumor cells and present antigens associated with tumor cells $(12,16)$. Although DCs alone did not induce reproducible anti-tumor effects on the untreated tumor side, the combination of telomelysin treatment and administration of intratumoral DCs produced stronger antitumor effects on remote tumors. We hypothesize that the increased apoptotic tumor cells, following treatment with telomelysin, release tumor antigens that are taken up by DCs.

In contrast, Huang et al and other researchers reported that telomelysin was transported to the untreated tumor through the bloodstream and then sprinted within the xenograft tumor in nude mice $(8,14,17)$. In this study, we analyzed an immunocomplete mouse model. Therefore, the anti-tumor activity of telomelysin on the untreated side may not only be a direct effect of the bloodstream but also an immunological activity induced by increasing the source of tumor antigens.
In the present study, we successfully demonstrated the anti-tumor effects of telomelysin in vitro and in vivo. Telomelysin may be applied for the therapy of advanced HNSCC patients with multiple tumors, especially multiple metastases.

\section{References}

1. Landis SH, Murray T, Bolden S and Wingo PA: Cancer statistics. CA Cancer J Clin 49: 8-31, 31, 1999.

2. Forastiere AA, Goepfert H, Maor M, et al: Concurrent chemotherapy and radiotherapy for organ preservation in advanced laryngeal cancer. N Engl J Med 349: 2091-2098, 2003.

3. Blackburn EH: Structure and function of telomeres. Nature 350: 569-573, 1991.

4. Kim NW, Piatyszek MA, Prowse KR, et al: Specific association of human telomerase activity with immortal cells and cancer. Science 266: 2011-2015, 1994

5. Shay JW and Wright WE: Telomerase activity in human cancer. Curr Opin Oncol 8: 66-71, 1996.

6. Nakayama J, Tahara H, Tahara E, et al: Telomerase activation by hTRT in human normal fibroblasts and hepatocellular carcinomas. Nat Genet 18: 65-68, 1998.

7. Umeoka T, Kawashima T, Kagawa S, et al: Visualization of intrathoracically disseminated solid tumors in mice with optical imaging by telomerase-specific amplification of a transferred green fluorescent protein gene. Cancer Res 64: 6259-6265, 2004.

8. Kawashima T, Kagawa S, Kobayashi N, et al: Telomerasespecific replication-selective virotherapy for human cancer. Clin Cancer Res 10: 285-292, 2004.

9. Watanabe T, Hioki M, Fujiwara T, et al: Histone deacetylase inhibitor FR901228 enhances the antitumor effect of telomerase-specific replication-selective adenoviral agent OBP-301 in human lung cancer cells. Exp Cell Res 312: 256-265, 2006.

10. Taki M, Kagawa S, Nishizaki M, et al: Enhanced oncolysis by a tropism-modified telomerase-specific replication-selective adenoviral agent OBP-405 ('Telomelysin-RGD'). Oncogene 24: 3130-3140, 2005

11. Deng J, Kloosterbooer F, Xia W and Hung MC: The NH(2)terminal and conserved region 2 domains of adenovirus E1A mediate two distinct mechanisms of tumor suppression. Cancer Res 62: 346-350, 2002.

12. Albert ML, Sauter B and Bhardwaj N: Dendritic cells acquire antigen from apoptotic cells and induce class I-restricted CTLs. Nature 392: 86-89, 1998.

13. Endo Y, Sakai R, Ouchi M, et al: Virus-mediated oncolysis induces danger signal and stimulates cytotoxic T-lymphocyte activity via proteasome activator upregulation. Oncogene 27: 2375-2381, 2008

14. Kishimoto H, Kojima T, Watanabe Y, et al: In vivo imaging of lymph node metastasis with telomerase-specific replicationselective adenovirus. Nat Med 12: 1213-1219, 2006.

15. Larmonier N, Merino D, Nicolas A, et al: Apoptotic, necrotic, or fused tumor cells: an equivalent source of antigen for dendritic cell loading. Apoptosis 11: 1513-1524, 2006.

16. Fadok VA, Bratton DL, Rose DM, Pearson A, Ezekewitz RA and Henson PM: A receptor for phosphatidylserine-specific clearance of apoptotic cells. Nature 405: 85-90, 2000.

17. Huang P, Watanabe M, Kaku H, et al: Direct and distant antitumor effects of a telomerase-selective oncolytic adenoviral agent, OBP-301, in a mouse prostate cancer model. Cancer Gene Ther 15: 315-322, 2008. 